\title{
Transitions of Care for Postoperative Opioid Prescribing in Previously Opioid-Naïve Patients in the USA: a Retrospective Review
}

\author{
Michael P. Klueh, BS ${ }^{7}$, Hsou M. Hu, $P h D^{7}$, Ryan A. Howard, $M D^{7}$, Joceline V. Vu, MD' , \\ Calista M. Harbaugh, MD' , Pooja A. Lagisetty, MD' ${ }^{2,3}$, Chad M. Brummett, MD ${ }^{4}$, \\ Michael J. Englesbe, $M D^{1,5}$, Jennifer F. Waljee, MD, MPH, MS ${ }^{7}$, and Jay S. Lee, $M D^{7}$
}

'Department of Surgery, University of Michigan, Ann Arbor, MI, USA; ${ }^{2}$ Department of Internal Medicine, University of Michigan, Ann Arbor, MI, USA;
3VA Center for Clinical Management Research, VA Ann Arbor Healthcare System, Ann Arbor, MI, USA; ${ }^{4}$ Department of Anesthesiology, University of
Michigan, Ann Arbor, MI, USA; ${ }^{5}$ Section of Transplant Surgery, University of Michigan Health System, Ann Arbor, MI, USA.

BACKGROUND: New persistent opioid use is a common postoperative complication, with $6 \%$ of previously opioidnaïve patients continuing to fill opioid prescriptions 36 months after surgery. Despite these risks, it is unknown which specialties prescribe opioids to these vulnerable patients.

OBJECTIVE: To identify specialties prescribing opioids to surgical patients who develop new persistent opioid use.

DESIGN, SETTING, AND PARTICIPANTS: Using a national dataset of insurance claims, we identified opioidnaïve patients aged 18-64 years undergoing surgical procedures (2008-2014) who continued filling opioid prescriptions 3 to 6 months after surgery. We then examined opioid prescriptions claims during the 12 months after surgery, and identified prescribing physician specialty using National Provider Identifier codes.

MAIN MEASURES: Percentage of opioid prescriptions provided by each specialty evaluated at 90-day intervals during the 12 months after surgery.

KEY RESULTS: We identified 5276 opioid-naïve patients who developed new persistent opioid use. During the first 3 months after surgery, surgeons accounted for $69 \%$ of opioid prescriptions, primary care physicians accounted for $13 \%$, Emergency Medicine accounted for 2\%, Physical Medicine \& Rehabilitation (PM\&R)/Pain Medicine accounted for $1 \%$, and all other specialties accounted for $15 \%$. In contrast, 9 to 12 months after surgery, surgeons accounted for only $11 \%$ of opioid prescriptions, primary care physicians accounted for 53\%, Emergency Medicine accounted for 5\%, PM\&R/Pain Medicine accounted for $6 \%$, and all other specialties provided $25 \%$.

CONCLUSIONS: Among surgical patients who developed new persistent opioid use, surgeons provide the majority of opioid prescriptions during the first 3 months after surgery. By 9 to 12 months after surgery, however, the majority of opioid prescriptions were provided by primary care physicians. Enhanced care coordination between surgeons and primary care physicians could allow earlier

Electronic supplementary material The online version of this article (https://doi.org/10.1007/s11606-018-4463-1) contains supplementary material, which is available to authorized users.

Received November 9, 2017

Revised March 8, 2018

Accepted April 16, 2018

Published online June 11, 2018 identification of patients at risk for new persistent opioid use to prevent misuse and dependence.

KEY WORDS: care transitions; postoperative care; patient-centered outcomes research; surgery; health services research; surgery.

J Gen Intern Med 33(10):1685-91

DOI: $10.1007 / \mathrm{s} 11606-018-4463-1$

(c) Society of General Internal Medicine 2018

\section{INTRODUCTION}

Exposure to opioids is ubiquitous in surgical care in the USA, and overprescribing of opioids is common following surgical procedures. ${ }^{1-3}$ Moreover, exposure to opioid medications for any cause can result in long-term opioid use, ${ }^{4}$ and $3-7 \%$ of opioid-naïve patients are at risk for developing new persistent opioid use following elective surgery. ${ }^{5-8}$ Thus, millions of Americans each year are continuing opioid use beyond the normal recovery period of 90 days after a surgical procedure. ${ }^{9}$ Both overprescribing and new persistent opioid use have contributed to the national opioid crisis, which results in 91 overdose deaths per day. ${ }^{10}$ In efforts to address this public health concern, many states have adopted or are considering new legislature that limits initial opioid prescriptions for acute causes such as surgery. ${ }^{11}$

Despite these risks, it is unclear how patients with new persistent opioid use receive care during the acute surgical period. While $50 \%$ of all opioid prescriptions are prescribed by primary care clinicians and pain specialists, ${ }^{12}$ their role during the postoperative period remains unknown. Identifying which physician specialties prescribe opioids to patients with new persistent opioid use is critical to forming multidimensional care strategies for early interventions aimed at preventing chronic opioid use, reducing opioid misuse, and preventing excess opioids entering communities.

Within this context, we examined a national retrospective cohort of patients undergoing surgical procedures to identify specialties prescribing opioids to surgical patients who developed new persistent opioid use. We evaluated the percentage of opioid prescriptions provided by each specialty at 90-day time intervals during the twelve months after surgery. We hypothesized that surgeons account for the majority of opioid 
prescriptions earlier in the postoperative period, while primary care clinicians account for the majority of opioid prescriptions later in the postoperative period.

\section{METHODS}

We conducted a nationwide retrospective study on a cohort of US adults using insurance claims data. The University of Michigan Institutional Review Board approved the exempt status of this study as all data was de-identified (HUM00118524).

\section{Data Source and Cohort}

We examined insurance claims data from Optum Clinformatics Data Mart, a large national database that includes commercial health plan data from all 50 states, the District of Columbia, and Puerto Rico. Prior to inclusion to the database, inpatient, outpatient, and pharmacy claims submitted for payment are verified and de-identified.

In this retrospective study, our study period spanned from January 1, 2007, to March 31, 2015, to include US adults aged 18-64 that underwent common surgical procedures between January 1, 2008, and March 31, 2014, and filled an opioid prescription attributed to surgery. This accounted for our 12month preoperative and postoperative follow-up. In accordance with previous work, ${ }^{5}$ we determined an opioid prescription was attributed to surgery if filled 30 days before to 14 days after surgery. To be included, patients must have maintained insurance coverage during the 12 months before and after their date of surgery. In an effort to minimize the inclusion of complications, we excluded any patients with hospital admissions greater than 30 days or a subsequent surgical procedure within 12 months after surgery. The latter was accomplished by excluding patients with an anesthesia Current Procedural Terminology (CPT) code within 1 year after surgery.

Surgical procedures included both minor and major surgeries. We limited surgeries to common procedures aligned with previous work. ${ }^{5}$ We excluded oncologic and orthopedic procedures as patients undergoing these procedures have higher incidence of persistent opioid use following surgery when compared to other common surgeries. ${ }^{13,14}$ Minor surgeries included laparoscopic cholecystectomy, laparoscopic appendectomy, hemorrhoidectomy, varicose vein removal, parathyroidectomy, thyroidectomy, transurethral prostate surgery, and carpal tunnel release. Major surgeries included bariatric surgery, ventral incisional hernia repair, reflux surgery, hysterectomy, and colectomy. These procedures were identified using Current Procedural Terminology (CPT) codes and International Statistical Classification of Diseases and Related Health Problems (ICD-9) procedure codes (Online Appendix).

\section{Definitions of Patient and Provider Categories}

Patients were identified by preoperative opioid use as previously described. ${ }^{5}$ Opioid-naïve patients with new persistent use did not fill any opioid prescriptions between 12 months to 31 days before surgery, filled an opioid prescription attributed to surgery, and then filled at least one additional opioid prescription between 91 and 180 days after surgery. Aligned with previous studies examining postoperative opioid use in opioid-naïve patients, ${ }^{5-8}$ this definition relies on a normal surgical recovery within 90 days after surgery. ${ }^{9}$

Provider taxonomy was derived from unique identification numbers assigned to healthcare providers. We used National Provider Identifier (NPI) codes to stratify providers into five groups: (1) Surgery; (2) Primary Care; (3) Cardiology, Gastroenterology, Oncology, Neurology, \& Other; (4) Physical Medicine \& Rehabilitation (PM\&R)/Pain Medicine; and (5) Emergency Medicine. Pain Medicine specialties included Anesthesiology as well as addiction and pain subspecialties. We chose to group PM\&R with Pain Medicine because both specialties frequently provide care to patients with chronic pain, ${ }^{15}$ and opioid prescriptions account for over $36 \%$ of all prescriptions provided by both these specialties. ${ }^{12}$ To prevent potential misclassification errors, we excluded physician assistants and nurse practitioners that we could not associate with a specialty using NPI codes.

\section{Outcomes}

Our primary outcome was the percentage of opioid prescriptions provided by each specialty during the twelve months after surgery evaluated at 90-day intervals.

\section{Statistical Analysis}

To compare patients who received opioid prescriptions from different specialties, we assigned each patient to a single specialty group based on the specialty that provided the first opioid prescription from 91 to 180 days after surgery. This was chosen because our definition of new persistent opioid use requires patients to fill at least one opioid prescription during this time period. ${ }^{5}$ We then compared patient characteristics among provider groups. Patient characteristics included gender, race, age, education, geographic region, surgery type, Charlson comorbidity index, tobacco use, mental disorders, and pain disorders. Chi-square test was used to test categorical variables and analysis of variance (ANOVA) tests were used to compare means among provider groups. $p$ values less than 0.05 were considered statistically significant.

Data Availability. The datasets generated and/or analyzed during the current study available from the corresponding author on reasonable request.

\section{RESULTS}

We identified 5276 opioid-naïve patients with new persistent opioid use that met the inclusion criteria, underwent a surgical procedure during the study period, and filled an opioid 
prescription attributed to surgery. Overall, a total of 20,830 opioid prescriptions were provided during the study period. Surgeons provided the most opioid prescriptions $(n=8118$; $39.0 \%$ ), while primary care provided 6101 opioid prescriptions (29.3\%). Cardiology, Gastroenterology, Oncology, Neurology, and Other provided 4708 opioid prescriptions (22.6\%). Emergency medicine provided 1464 opioid prescriptions (7.0\%), and the remaining 439 prescriptions were provided by PM\&R/Pain medicine (2.1\%).

Figure 1 illustrates the percentage of opioid prescriptions by provider specialty for opioid-naïve patients with new persistent use. During the first 3 months after surgery, surgeons accounted for $69 \%$ of opioid prescriptions, primary care physicians accounted for $13 \%$, Emergency Medicine accounted for 2\%, Physical Medicine \& Rehabilitation (PM\&R) / Pain Medicine accounted for $1 \%$, and all other specialties accounted for $15 \%$. Three to 6 months after surgery, surgeons provided for $20 \%$ of opioid prescriptions, primary care physicians accounted for $36 \%$, Emergency Medicine accounted for $11 \%$, PM\&R/Pain Medicine accounted for 2\%, and all other specialties provided $31 \%$. Six to nine months after surgery, surgeons accounted for $16 \%$ of opioid prescriptions, primary care physicians provided $48 \%$, Emergency Medicine provided 5\%, PM\&R/Pain Medicine accounted for 6\%, and all other specialties accounted for $26 \%$. Nine to 12 months after surgery, surgeons accounted for only $11 \%$ of opioid prescriptions, primary care physicians accounted for 53\%, Emergency Medicine accounted for 5\%, PM\&R / Pain Medicine accounted for $6 \%$, and all other specialties provided $25 \%$. A total 8226 opioid prescriptions were prescribed in the first 3 months after surgery, and opioid prescribing decreased to 6958 prescriptions 3 to 6 months after surgery. Fewer prescriptions were filled at later time points, with 2167 prescriptions six to nine months and 1963 prescriptions nine to 12 months after surgery.

As detailed in Table 1, we stratified patients by the specialty who provided the first opioid prescription 91-180 days after surgery in order to compare differences in patient characteristics among provider groups. There were no significant differences in patients' gender or race between provider groups. In all groups, opioid-naïve patients with new persistent opioid use were predominantly female, ranging from 69 to $75 \%$ female among provider groups $(p=0.137)$. Patients were also predominantly white, varying from 71 to $74 \%$ white between providers $(p=0.353)$. We did find significant differences with respect to patient age $(p<0.001)$. Emergency Medicine was more likely to provide opioid prescriptions to younger patients (mean 42.6 years; SD 0.4) while older patients were more likely to receive opioids from PM\&R/Pain Medicine (mean 47.1 years; SD 1.4). Patient education level differed significantly among provider groups $(p<0.001)$. For example, PM\&R/Pain Medicine was more likely to provide opioid prescriptions to patients with a Bachelor's degree or higher $(n=10 ; 17.5 \%)$. In contrast, Primary Care $(n=637 ; 36.0 \%)$ and Emergency Medicine $(n=254 ; 35.8 \%)$ were more likely to prescribe opioids to patients with a high school diploma.
Geographic region also showed a significant difference $(p<$ 0.001 ), although patients were more likely to reside in the South Atlantic region for all provider groups (26.3 to 32.7\%). Minor surgery was more prominent for all provider groups but significant differences were observed $(p=0.021)$. Notably, PM\&R/Pain Medicine was more likely to provide opioids to patients that underwent minor surgery $(n=50 ; 87.7 \%)$, and surgeons were more likely to prescribe opioids to patients following major surgical procedures $(n=279 ; 25.7 \%)$.

Pertinent past medical history is also shown in Table 1. There was a significant difference between provider groups with respect to patients' Charlson comorbidity index ( $p=$ 0.01 ). Emergency Medicine was more likely to provide opioid prescriptions to patients with fewer comorbidities $(0.9$; SD 0.1 ) while $P M \& R / P a i n$ Medicine was more likely to prescribe opioids to patients with increased comorbidities (1.3; SD 0.3). Significant differences were also seen in regard to tobacco use $(p<0.001)$. Tobacco use was most common among patients that received opioid prescriptions from primary care $(n=664$; $37.6 \%$ ) and least common in patients that receive opioids from surgeons $(n=312 ; 28.8 \%)$. Among mental disorders, significant differences were observed in anxiety disorder $(p<0.001)$, mood disorder $(p=0.003)$, schizophrenia and other psychotic disorders $(p=0.001)$, and drug and substance use disorders $(p=0.001)$. For example, primary care was more likely to prescribe opioids to patients with anxiety disorder $(n=394$; $22.3 \%$ ), while PM\&R/Pain Medicine was more likely to provide opioids to patients with mood disorder $(n=16 ; 28.1 \%)$. For pain disorders, we found significant differences with back pain $(p<0.001)$, neck pain $(p<0.001)$, arthritis $(p<0.001)$, and other pain disorders $(p<0.001)$. PM\&R/Pain Medicine was more likely to prescribe opioids to patients with these conditions (back pain $[n=44,77.2 \%]$; neck pain $[n=24$, $42.1 \%]$; arthritis $[n=50,87.7 \%]$; other pain disorders $[n=$ $39,68.4 \%]$ ). Conversely, Cardiology, Gastroenterology, Oncology, Neurology, and Other were least likely to prescribe opioids to patients with back pain $(n=463 ; 27.9 \%)$, neck pain $(n=256 ; 15.4 \%)$, and arthritis $(n=899 ; 54.2 \%)$.

\section{DISCUSSION}

Among opioid-naïve patients with new persistent opioid use following common surgical procedures, the majority of postoperative opioid prescribing transitioned from surgeons to primary care clinicians between 3 to 6 months after surgery. By 6 months after surgery, surgeons provided $20 \%$ or less of opioid prescriptions, and primary care clinicians increased to over $50 \%$ of prescriptions 12 months after surgery. Cardiology, Gastroenterology, Oncology, Neurology, and Other played a prominent role in postoperative opioid prescribing, providing over $25 \%$ of opioid prescriptions more than 6 months after surgery. During the transition of care period (3-6 months after surgery), PM\&R/Pain Medicine provided less than $2 \%$ of opioid prescriptions, while emergency medicine provided 


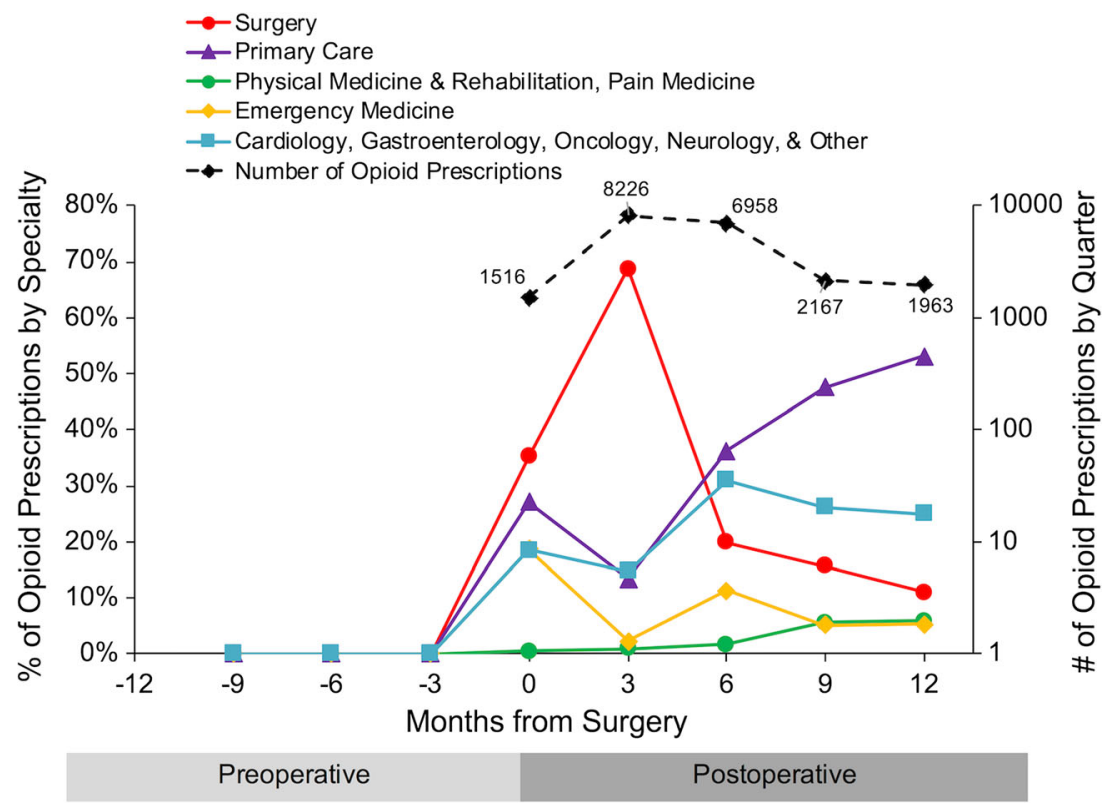

Fig. 1 Prescriber specialty for opioid prescriptions among patients with new persistent opioid use. Among opioid-naïve patients with new persistent opioid use, the majority of postoperative opioid prescribing transitioned from surgeons to primary care clinicians between 3 to 6 months after surgery. Cardiology, Gastroenterology, Oncology, Neurology, and Other provided 25-31\% of prescriptions beyond 3 months after surgery. PM\&R/Pain Medicine provided less than $6 \%$ of prescriptions in the study period. Emergency medicine reached $11 \%$ of prescriptions at 6 months after surgery. The highest amount of opioid prescriptions were prescribed in the first 3 months after surgery $(n=$ 8226).

$11 \%$ of opioid prescriptions during the transition. Notably, more than 3 months after surgery, surgeons were more likely than primary care to prescribe opioids to patients with a higher education level and fewer mental or pain disorders. Overall, our data suggests that the first 3 months after surgery is a critical time period for future research to determine if improving provider-to-provider communication between surgeons and subsequent opioid prescribers can improve care for opioid-naïve patients.

This was the first study to examine the transition of postoperative opioid-managed pain in terms of opioid prescriptions by provider specialty. More broadly, one study examined the nationwide percentages of opioid prescriptions by specialty for all patients, finding that $44.5 \%$ of prescriptions were provided by primary care clinicians, $9.8 \%$ by surgeons, $8.2 \%$ by PM\&R/Pain Medicine, and $4.3 \%$ by Emergency Medicine. ${ }^{12}$ In our study, surgeons expectedly played a greater role during the postoperative transition period for opioid-naïve patients with new persistent use, but these findings highlight the underutilization of pain specialists and overrepresentation of emergency medicine during the postoperative period.

Although not a primary outcome for our study, our unadjusted rates of new persistent use among previously opioid-naïve patients (6.3\%; data not shown) were consistent with previous work. $^{5-8}$ Opioid prescription refills and extended duration of opioid use after surgical procedures have been associated with increased opioid dependence, abuse, and overdose. ${ }^{16}$ Consequently, millions of Americans every year must confront the risk of opioid-related complications when exposed to opioids after surgery. Considering the increased healthcare costs and risk of mortality associated with opioid dependence, ${ }^{17-19}$ healthcare providers must reexamine current practices regarding postoperative opioid prescribing. When compared to national opioid prescribing rates, ${ }^{12}$ our data showed that Emergency Medicine provided over twice their national average of opioid prescriptions during the postoperative transition of care. Following the transition period, emergency medicine prescribing decreased by over $50 \%$ while primary care and PM\&R/Pain Medicine increased. These findings may suggest a lack of coordination between surgeons and subsequent providers regarding opioid-managed pain following surgery.

Improved transitions of care following surgery could result in more successful tapering of opioid use, but there are additional confounding barriers that must be addressed by future work. Geographic barriers, underinsurance, and referral practices limit access to pain specialists. ${ }^{20,21}$ Among opioid-naïve patients with new persistent use, we found that utilization of PM\&R/Pain Medicine for opioid prescriptions varied among geographic regions. Also seen in Table 1, PM\&R/Pain Medicine physicians were more likely to prescribe opioids following minor surgeries to older patients that have an education level of at least a bachelor degree, increased comorbidities, and pain disorders. Additionally, primary care clinicians and patients have expressed difficulty in tapering long-term opioid use. ${ }^{22-24}$ In this study, the transition of opioid prescribing to primary care occurred 3-6 months after surgery, and primary care continued to increase in percentage of opioid prescribing throughout the study period. Early and deliberate transition of care may be required for patients that continue to require opioids following surgery. Failing to successfully wean 
Table 1 Characteristics of Opioid-Naïve Patients with New Persistent Use by Provider Group

\begin{tabular}{|c|c|c|c|c|c|c|}
\hline & $\begin{array}{l}\text { Primary care } \\
(n=1767)\end{array}$ & $\begin{array}{l}\text { Surgery } \\
(n=1084)\end{array}$ & $\begin{array}{l}\text { Emergency } \\
\text { medicine } \\
(n=709)\end{array}$ & $\begin{array}{l}\text { PM\&R, pain } \\
\text { specialist } \\
(n=57)\end{array}$ & $\begin{array}{l}\text { Cardiology, } \\
\text { gastroenterology, } \\
\text { oncology, neurology, } \\
\text { and other }(n=1659)\end{array}$ & $p$ value $*$ \\
\hline & $\begin{array}{l}\text { No. cases } \\
\text { (\% total) }\end{array}$ & $\begin{array}{l}\text { No. cases } \\
\text { (\% total) }\end{array}$ & $\begin{array}{l}\text { No. cases } \\
\text { (\% total) }\end{array}$ & $\begin{array}{l}\text { No. cases } \\
\text { (\% total) }\end{array}$ & $\begin{array}{l}\text { No. cases } \\
\text { (\% total) }\end{array}$ & \\
\hline \multicolumn{6}{|l|}{ Gender } & 0.137 \\
\hline Female & $1225(69.3 \%)$ & $808(74.5 \%)$ & $495(69.8 \%)$ & $40(70.2 \%)$ & $1158(69.8 \%)$ & \\
\hline \multicolumn{6}{|l|}{ Race } & 0.353 \\
\hline White & $1250(70.7 \%)$ & $773(71.3 \%)$ & $510(71.9 \%)$ & $42(73.7 \%)$ & $1187(71.5 \%)$ & \\
\hline Black & $253(14.3 \%)$ & $128(11.8 \%)$ & $89(12.6 \%)$ & $5(8.8 \%)$ & $222(13.4 \%)$ & \\
\hline Hispanic & $174(9.8 \%)$ & $119(11.0 \%)$ & $70(9.9 \%)$ & $4(7.0 \%)$ & $156(9.4 \%)$ & \\
\hline Others/unknown & $90(5.1 \%)$ & $64(5.9 \%)$ & $40(5.6 \%)$ & $6(10.5 \%)$ & $94(5.7 \%)$ & \\
\hline Mean age (SD) & $46.2(0.3)$ & $45.5(0.3)$ & $42.6(0.4)$ & $47.7(1.4)$ & $44.6(0.3)$ & $<0.001$ \\
\hline \multicolumn{6}{|l|}{ Education } & $<0.001$ \\
\hline Less than 12th grade & $9(0.5 \%)$ & $8(0.7 \%)$ & $1(0.1 \%)$ & $0(0.0 \%)$ & $8(0.5 \%)$ & \\
\hline High school diploma & $637(36.0 \%)$ & $327(30.2 \%)$ & $254(35.8 \%)$ & $13(22.8 \%)$ & $479(28.9 \%)$ & \\
\hline Less than bachelor degree & $916(51.8 \%)$ & $580(53.5 \%)$ & $373(52.6 \%)$ & $32(56.1 \%)$ & $913(55.0 \%)$ & \\
\hline Bachelor degree plus & $186(10.5 \%)$ & $156(14.4 \%)$ & $75(10.6 \%)$ & $10(17.5 \%)$ & $243(14.6 \%)$ & \\
\hline Unknown & $19(1.1 \%)$ & $13(1.2 \%)$ & $6(0.8 \%)$ & $2(3.5 \%)$ & $16(1.0 \%)$ & \\
\hline \multicolumn{6}{|l|}{ Geographic region } & $<0.001$ \\
\hline East North Central & $297(16.8 \%)$ & $175(16.1 \%)$ & $104(14.7 \%)$ & $6(10.5 \%)$ & $224(13.5 \%)$ & \\
\hline East South Central & $105(5.9 \%)$ & $63(5.8 \%)$ & $33(4.7 \%)$ & $3(5.3 \%)$ & $95(5.7 \%)$ & \\
\hline Middle Atlantic & $54(3.1 \%)$ & $38(3.5 \%)$ & $22(3.1 \%)$ & $6(10.5 \%)$ & $70(4.2 \%)$ & \\
\hline Mountain & $147(8.3 \%)$ & $78(7.2 \%)$ & $54(7.6 \%)$ & $7(12.3 \%)$ & $180(10.8 \%)$ & \\
\hline New England & $38(2.2 \%)$ & $23(2.1 \%)$ & $21(3.0 \%)$ & $0(0.0 \%)$ & $41(2.5 \%)$ & \\
\hline Pacific & $97(5.5 \%)$ & $62(5.7 \%)$ & $37(5.2 \%)$ & $3(5.3 \%)$ & $74(4.5 \%)$ & \\
\hline South Atlantic & $529(29.9 \%)$ & $301(27.8 \%)$ & $232(32.7 \%)$ & $15(26.3 \%)$ & $488(29.4 \%)$ & \\
\hline West North Central & $156(8.8 \%)$ & $81(7.5 \%)$ & $80(11.3 \%)$ & $2(3.5 \%)$ & $114(6.9 \%)$ & \\
\hline West South Central & $342(19.4 \%)$ & $262(24.2 \%)$ & $125(17.6 \%)$ & $14(24.6 \%)$ & $371(22.4 \%)$ & \\
\hline Unknown & $2(0.1 \%)$ & $1(0.1 \%)$ & $1(0.1 \%)$ & $1(1.8 \%)$ & $2(0.1 \%)$ & \\
\hline \multicolumn{6}{|l|}{ Surgery type $†$} & 0.021 \\
\hline Major & $438(24.8 \%)$ & $279(25.7 \%)$ & $145(20.5 \%)$ & $7(12.3 \%)$ & $403(24.3 \%)$ & \\
\hline Minor & $1329(75.2 \%)$ & $805(74.3 \%)$ & $564(79.5 \%)$ & $50(87.7 \%)$ & $1256(75.7 \%)$ & \\
\hline Mean Charlson comorbidity index (SD) & $1.1(0.0)$ & $1.0(0.1)$ & $0.9(0.1)$ & $1.3(0.3)$ & $1.1(0.0)$ & 0.010 \\
\hline Tobacco use & $664(37.6 \%)$ & $312(28.8 \%)$ & $235(33.1 \%)$ & $21(36.8 \%)$ & $525(31.6 \%)$ & $<0.001$ \\
\hline \multicolumn{7}{|l|}{ Mental disorders } \\
\hline Disruptive disorder & $56(3.2 \%)$ & $17(1.6 \%)$ & $20(2.8 \%)$ & $2(3.5 \%)$ & $20(1.2 \%)$ & 0.114 \\
\hline Adjustment disorder & $87(4.9 \%)$ & $43(4.0 \%)$ & $31(4.4 \%)$ & $3(5.3 \%)$ & $86(5.2 \%)$ & 0.638 \\
\hline Anxiety disorder & $394(22.3 \%)$ & $175(16.1 \%)$ & $133(18.8 \%)$ & $11(19.3 \%)$ & $269(16.2 \%)$ & $<0.001$ \\
\hline Mood disorder & $434(24.6 \%)$ & $212(19.6 \%)$ & $149(21.0 \%)$ & $16(28.1 \%)$ & $331(20.0 \%)$ & 0.003 \\
\hline Suicide and intentional self-inflicted injury & $13(0.7 \%)$ & $4(0.4 \%)$ & $2(0.3 \%)$ & $0(0.0 \%)$ & $8(0.5 \%)$ & 0.520 \\
\hline Personality disorders & $11(0.6 \%)$ & $7(0.6 \%)$ & $2(0.3 \%)$ & $0(0.0 \%)$ & $6(0.4 \%)$ & 0.622 \\
\hline Schizophrenia and other psychotic & $21(1.2 \%)$ & $11(1.0 \%)$ & $5(0.7 \%)$ & $3(5.3 \%)$ & $7(0.4 \%)$ & 0.001 \\
\hline \multicolumn{7}{|l|}{ disorders } \\
\hline Drug and substance use disorder & $74(4.2 \%)$ & $22(2.0 \%)$ & $24(3.4 \%)$ & $4(7.0 \%)$ & $37(2.2 \%)$ & 0.001 \\
\hline Other mental disorders & $94(5.3 \%)$ & $51(4.7 \%)$ & $30(4.2 \%)$ & $4(7.0 \%)$ & $50(3.0 \%)$ & 0.014 \\
\hline \multicolumn{7}{|l|}{ Pain disorders } \\
\hline Back pain & $830(47.0 \%)$ & $345(31.8 \%)$ & $289(40.8 \%)$ & $44(77.2 \%)$ & $463(27.9 \%)$ & $<0.001$ \\
\hline Neck pain & $388(22.0 \%)$ & $190(17.5 \%)$ & $165(23.3 \%)$ & $24(42.1 \%)$ & $256(15.4 \%)$ & $<0.001$ \\
\hline Arthritis & $1151(65.1 \%)$ & $628(57.9 \%)$ & $430(60.6 \%)$ & $50(87.7 \%)$ & $899(54.2 \%)$ & $<0.001$ \\
\hline Other pain disorders & $996(56.4 \%)$ & $506(46.7 \%)$ & $396(55.9 \%)$ & $39(68.4 \%)$ & $811(48.9 \%)$ & $<0.001$ \\
\hline
\end{tabular}

*Chi-square test was used to test categorical variables; ANOVA (analysis of variance) tests were used to compare means

+ See Online Appendix for minor and major surgeries included

opioid-naïve patients in the immediate postoperative period could lead to clinical inertia, ${ }^{25}$ where prescribing continues without efforts to taper opioids.

For patients struggling to taper opioids after surgery, more work is needed to determine if early coordination between surgeons and primary care clinicians can alter these patients' clinical trajectories. Perioperative patient education should include realistic expectations regarding postoperative pain and duration of opioid use following surgery. Providers can also reduce postoperative opioid requirements by utilizing non-opioid analgesics, such as acetaminophen or NSAIDs. ${ }^{26-28}$ Shorter initial opioid prescriptions after surgery would trigger a feedback loop between patient and physician, allowing surgeons to rapidly identify patients that continue to require opioids. ${ }^{29,30}$ Heightened awareness among patients, surgeons, and primary care physicians that surgery increases the risk of new persistent opioid use is necessary to promote improved communication and aggressive tapering of opioids while still in the acute surgical period. When practical, referring patients with persistent opioid use after surgery to pain specialists may prove useful in optimizing patient care. ${ }^{31,32}$

This study has limitations to address. First, using retrospective insurance claims data placed our study at risk for misclassification error as we used National Provider Identifier codes 
to group provider specialties for clarity. We attempted to address this concern by excluding physician assistants and nurse practitioners that were not associated with a specialty. These providers represented a small proportion in our study, prescribing fewer than 800 opioid prescriptions. However, they play an important role and should factor into future analyses. Additionally, some non-primary care clinicians may manage prescribing medications for certain medical conditions (e.g. rheumatology for rheumatoid arthritis). We do not currently have data describing which patients are more likely to transition to primary care versus another specialty, but this is the focus of ongoing work. Second, we cannot discern patients' motivation to receive opioid prescriptions or glean actual opioid consumption using claims data. Also, prescribing rates do not identify mean daily doses or prescription size. Increasing national focus on the opioid crisis has prompted efforts on reducing opioid utilization through new regulations and legislation, which may have subsequently altered practice patterns after the finishing point of our study period. Finally, our cohort was selected from insured US adults, and this data may not be applicable to under- or uninsured patients or to patients younger than 18 or older than 64 years.

Among surgical patients who develop new persistent opioid use, the majority of postoperative opioid prescribing transitioned from surgeons to primary care clinicians between 3 to 6 months after surgery. Further work is needed to determine if enhanced care coordination between patients, surgeons, and primary care clinicians could allow identification of risk for persistent opioid use to prevent opioid misuse and dependence.

Prior Presentations: This work has not been previously published or presented.

Research Support: Dr. Lee is a National Research Service Award postdoctoral fellow supported by the National Cancer Institute (5T32 CA009672-23). Drs. Brummett, Englesbe, and Waljee receive funding from the Michigan Department of Health and Human Services. Mr. Klueh is supported by the National Institutes of Health's T35 ShortTerm Training Grant for Medical Students (5T35 HL007690-34). The content of this study is solely the responsibility of the authors and does not necessarily represent the official views of the National Institutes of Health or the Michigan Department of Health and Human Services.

Corresponding Author: Michael J. Englesbe, MD; Section of Transplant Surgery University of Michigan Health System, Ann Arbor, MI, USA (e-mail: englesbe@med.umich.edu).

Contributors Significant contributions to the work were completed by the authors. All authors had full access to the study data, take responsibility for the accuracy of the analysis, and approve the submission of this manuscript.

Funding Dr. Brummett is a consultant for Recro Pharma (Malvern, PA) and Heron Pharm; not related to the present work. Dr. Brummett receives research funding from Neuros Medical Inc. (Willoughby Hills, Ohio). Dr. Englesbe receives research funding from the Substance Abuse and Mental Health Services Administration. Dr. Waljee receives research funding from the Agency for Healthcare Research and Quality (KO8 1K08HSO23313-01), the American College of Surgeons, and the American Foundation for Surgery of the Hand. Dr. Waljee is an unpaid consultant for 3M Health Information systems.

\section{Compliance with ethical standards:}

Conflict of interest: The authors declare that they have no conflict of interest.

\section{REFERENCES}

1. Thiels CA, Anderson SS, Ubl DS, et al. Wide variation and overprescription of opioids after elective surgery. Ann Surg. 2017.

2. Hill MV, McMahon ML, Stucke RS, Barth RJ, Jr. Wide variation and excessive dosage of opioid prescriptions for common general surgical procedures. Ann Surg. 2017;265(4):709-714.

3. Bates C, Laciak R, Southwick A, Bishoff J. Overprescription of postoperative narcotics: a look at postoperative pain medication delivery, consumption and disposal in urological practice. $J$ Urol. 2011;185(2):551-555.

4. Shah A, Hayes CJ, Martin BC. Factors influencing long-term opioid use among opioid naive patients: an examination of initial prescription characteristics and pain etiologies. J Pain. 2017.

5. Brummett CM, Waljee JF, Goesling $\mathbf{J}$, et al. New persistent opioid use after minor and major surgical procedures in US adults. JAMA Surg. 2017;152(6):e170504.

6. Clarke H, Soneji N, Ko DT, Yun L, Wijeysundera DN. Rates and risk factors for prolonged opioid use after major surgery: population based cohort study. BMJ. 2014;348:g1251.

7. Soneji N, Clarke HA, Ko DT, Wijeysundera DN. Risks of developing persistent Opioid use after major surgery. JAMA Surg. 2016;151(11):1083-1084.

8. Alam A, Gomes T, Zheng H, Mamdani MM, Juurlink DN, Bell CM. Long-term analgesic use after low-risk surgery: a retrospective cohort study. Arch Intern Med. 2012;172(5):425-430.

9. Kehlet H, Rathmell JP. Persistent postsurgical pain: the path forward through better design of clinical studies. Anesthesiology. 2010;112(3):514-515.

10. Rudd RA, Seth P, David F, Scholl L. Increases in drug and opioidinvolved overdose deaths-United States, 2010-2015. MMWR Morb Mortal Wkly Rep. 2016;65(5051):1445-1452.

11. Baker-White A. A look at state legislation limiting opioid prescriptions. 2017; http://www.astho.org/StatePublicHealth/A-Look-at-State-Legislation-Limiting-Opioid-Prescriptions/2-23-17/. Accessed March 22, 2018.

12. Levy B, Paulozzi L, Mack KA, Jones CM. Trends in opioid analgesicprescribing rates by specialty, U.S., 2007-2012. Am J Prev Med. 2015;49(3):409-413.

13. Goesling J, Moser SE, Zaidi B, et al. Trends and predictors of opioid use after total knee and total hip arthroplasty. Pain. 2016;157(6):1259-1265.

14. Lee JS, Hu HM, Edelman AL, et al. New persistent opioid use among patients with Cancer after curative-intent surgery. J Clin Oncol. 2017;35(36):4042-4049.

15. Porucznik CA, Johnson EM, Rolfs RT, Sauer BC. Specialty of prescribers associated with prescription opioid fatalities in Utah, 20022010. Pain Med. 2014;15(1):73-78.

16. Brat GA, Agniel D, Beam A, et al. Postsurgical prescriptions for opioid naive patients and association with overdose and misuse: retrospective cohort study. BMJ. 2018;360:j5790.

17. Bohnert AS, Valenstein M, Bair MJ, et al. Association between opioid prescribing patterns and opioid overdose-related deaths. JAMA. 2011;305(13): 1315-1321

18. Leider HL, Dhaliwal J, Davis EJ, Kulakodlu M, Buikema AR. Healthcare costs and nonadherence among chronic opioid users. Am J Manag Care. 2011;17(1):32-40.

19. Ray WA, Chung CP, Murray KT, Hall K, Stein CM. Prescription of longacting opioids and mortality in patients with chronic noncancer pain. JAMA. 2016;315(22):2415-2423.

20. Ho D, Imai K, King G, Stuart EA. MatchIt: nonparametric preprocessing for parametric causal inference. J Stat Softw. 2011;42(8):28.

21. Eaton LH, Gordon DB, Wyant S, et al. Development and implementation of a telehealth-enhanced intervention for pain and symptom management. Contemp Clin Trials. 2014;38(2):213-220.

22. Penney LS, Ritenbaugh C, DeBar LL, Elder C, Deyo RA. Provider and patient perspectives on opioids and alternative treatments for managing chronic pain: a qualitative study. BMC Fam Pract. 2017;17(1):164.

23. Frank JW, Levy C, Matlock DD, et al. Patients' perspectives on tapering of chronic opioid therapy: a qualitative study. Pain Med. 2016;17(10):1838-1847. 
24. Hao J, Lucido D, Cruciani RA. Potential impact of abrupt opioid therapy discontinuation in the management of chronic pain: a pilot study on patient perspective. J Opioid Manag. 2014;10(1):9-20.

25. Phillips LS, Branch WT, Cook CB, et al. Clinical inertia. Ann Intern Med. 2001;135(9):825-834.

26. Hill MV, Stucke RS, McMahon ML, Beeman JL, Barth RJ, Jr. An educational intervention decreases opioid prescribing after general surgical operations. Ann Surg. 2017.

27. Hah JM, Bateman BT, Ratliff J, Curtin C, Sun E. Chronic opioid use after surgery: implications for perioperative management in the face of the opioid epidemic. Anesth Analg. 2017;125(5):1733-1740.
28. Kumar K, Kirksey MA, Duong S, Wu CL. A review of opioid-sparing modalities in perioperative pain management: methods to decrease opioid use postoperatively. Anesth Analg. 2017;125(5):1749-1760.

29. Dowell D, Haegerich TM, Chou R. CDC guideline for prescribing opioids for chronic pain-United States, 2016. JAMA. 2016;315(15):1624-1645.

30. Cifuentes M, Powell R, Webster B. Shorter time between opioid prescriptions associated with reduced work disability among acute low back pain opioid users. J Occup Environ Med. 2012;54(4):491-496.

31. Thomas DA, Chang D, Zhu R, Rayaz H, Vadivelu N. Concept of the ambulatory pain physician. Curr Pain Headache Rep. 2017;21(1):7.

32. Peppin JF, Cheatle MD, Kirsh KL, McCarberg BH. The complexity model: a novel approach to improve chronic pain care. Pain Med. 2015;16(4):653-666. 